Some measurements of the stresses produced at the surfaces of glass by grinding with loose abrasives

This content has been downloaded from IOPscience. Please scroll down to see the full text.

1922 Trans. Opt. Soc. 23170

(http://iopscience.iop.org/1475-4878/23/3/303)

View the table of contents for this issue, or go to the journal homepage for more

Download details:

IP Address: 164.76.7.55

This content was downloaded on 07/05/2017 at 08:01

Please note that terms and conditions apply.

You may also be interested in:

The structure of abraded glass surfaces

F W Preston

The stress conditions surrounding a diamond cut in glass

A J Dalladay and F Twyman

Variation in refractive index near the surfaces of glass melts

F Twyman and A J Dalladay

The nature of the polishing operation

F W Preston

More Notes on Glass Grinding and Polishing

James Weir French

The Grading of Carborundum for Optical Purposes

James Weir French

Comparison of the structure of sand-blasted and ground glass surfaces

F W Preston

On stresses in transparent materials as revealed by polarised light

E G Coker

Rubber models of yarns and cords; the 'doubling' of single rods

$\mathrm{N}$ Wilson and L R G Treloar 


\title{
SOME MEASUREMENTS OF THE STRESSES PRODUCED AT THE SURFACES OF GLASS BY GRINDING WITH LOOSE ABRASIVES
}

\author{
By A. J. DALLADAY, A.Inst.P. \\ (Communicated by Mr F. Twyman.)
}

MS. received, 2oth February, 1922. Read and discussed, gth March, I922.

THE object of this work, which was carried out five years ago in the laboratories of Messrs Adam Hilger, Ltd., was to ascertain what was the actual value of the compression in the grey surfaces produced on glass by grinding with the abrasives in general use, viz. carborundum, corundum and Naxos emery, in various degrees of fineness. The grey was in each case produced by first grinding out with the abrasive under test any sign of the pits produced by the previous grade, and then finishing the surface with fresh abrasive, taking care not to work it down.

The specimen of glass used was Hard Crown, $\mu_{D}=\mathrm{I}_{5} 5 \mathrm{I} 8$, and was in the form of a flat strip of the following dimensions:

$$
\begin{aligned}
& \text { Length } \quad l=76 \mathrm{~mm} . \\
& \text { Width } \quad b=\mathrm{Ir} \cdot 3 \mathrm{~mm} . \\
& \text { Thickness } a=3.24 \text { to } 3.035 \mathrm{~mm} \text {. at various stages of grey. } \\
& \text { Weight } \quad w=7.0 \mathrm{gm} .
\end{aligned}
$$

All faces were polished except the two small end faces.

The strip was supported at $45^{\circ}$ with the vertical and observed along its width with vertically polarised light, crossed nicols and a sensitive one-wave mica plate. No sign of double refraction could be detected. One side of the strip was then ground with No. 80 carborundum, and again observed. The strip now appeared stressed as if it were being subjected to pure bending combined with extension; i.e. the neutral axis was straight, and nearer the polished than the grey surface; that part of the strip adjacent to the grey surface was in tension, the other being in compression.

It was assumed that this stress system was the result of a uniform compression in the grey surface itself, which, although intense, was so closely confined to the grey surface that it could not be directly observed by polarised light. The method adopted to measure this compression was to balance the stresses at a point in the glass by applying a known bending moment, and from this to calculate the value of the total thrust in the grey layer. The bending moment necessary to bring about this balance proved to be $125 \mathrm{gm} . \mathrm{cm}$.

It can easily be shown that in a strip bent, as this one is, by a thrust in one surface, the neutral axis is shifted so that it is two-thirds of the distance from the stressed to the free surface; and from this it follows that if $F$ is the thrust per unit 


\section{Measurements of Stresses produced at the Surfaces of Glass I 71}

breadth in the stressed surface, and $M$ is the bending moment produced, $F=\mathbf{2} M / a b$, $a$ and $b$ being the thickness and width of the strip, as before. This gives a value for the thrust of $680 \mathrm{gm}$. weight, or $6.7 \times 10^{5}$ dynes, per unit breadth of surface.

No accurate measurements were made of the maximum double refraction present. These would have been difficult to make with any accuracy, and besides having no immediate bearing on the problem in hand, they can be deduced from the absolute value of the maximum stress present. The maximum double refraction close to the grey surface was probably about $\cdot 05 \lambda$ retardation.

Having made the measurements on the coarse carborundum surface, the specimen was taken out and reground with No. 180 carborundum. The measurements were then repeated, giving a new value for the thrust in the grey surface. The results of these measurements are tabulated and plotted below.

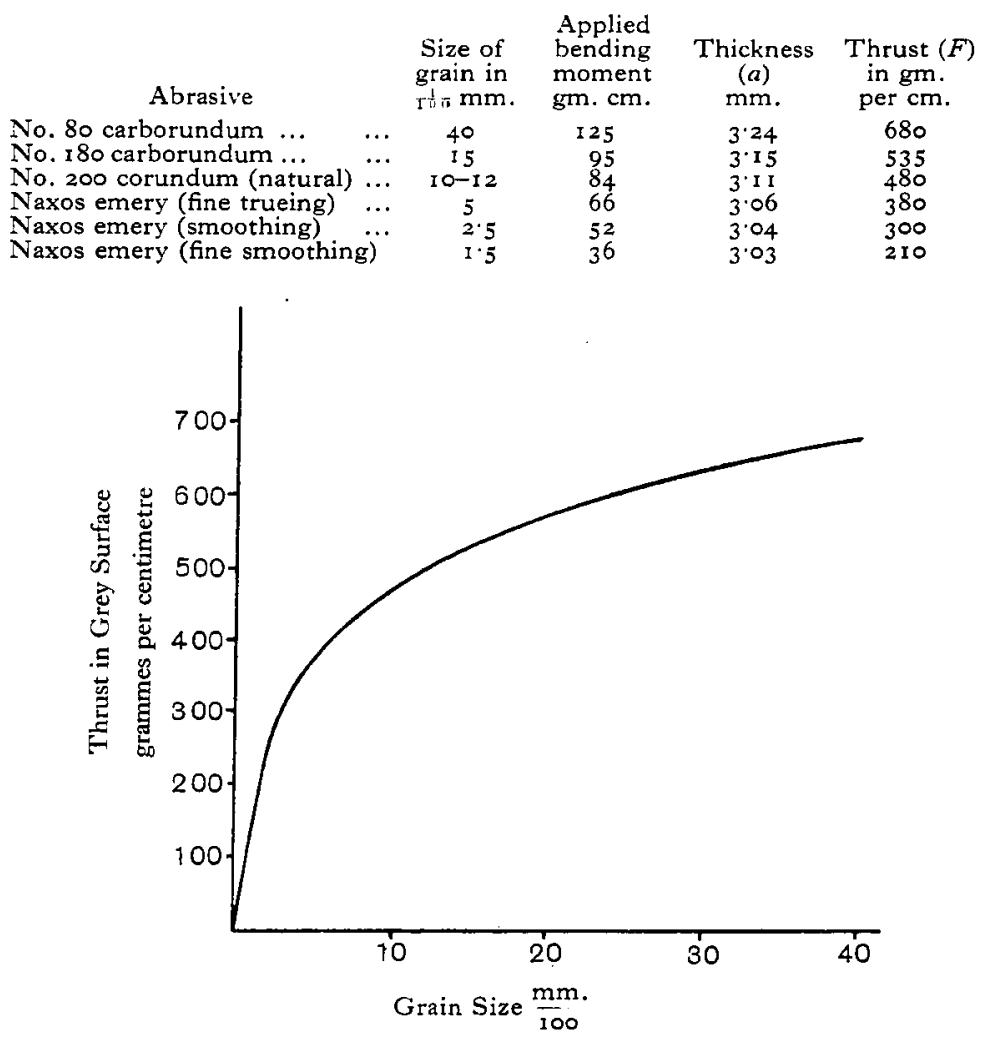

It will be seen that as the coarseness of grain increases the thrust increases, at first in linear proportion, but very much more slowly as the coarser abrasives are used, so that although the coarse carborundum was nearly three times the grain size of the fine carborundum, the thrust it produced was less than $4 / 3$ that 
produced by the fine. It should also be noticed that there is apparently no discontinuity as a result of changing from carborundum to natural corundum and thence to ordinary Naxos emery.

The determinations of the balancing bending moments could be made with fair accuracy. The differences between successive determinations with the same grey did not exceed 5 per cent. in the coarșe greys and ro per cent. in the finest. It might at first be thought that the unsymmetrical position of the neutral axis would cause an error in estimating the balancing point but it will be seen that the errors produced above and below the neutral axis are in opposite sense, and therefore destroy one another. The results are the mean of three determinations and no greater accuracy is justified in view of the uncertain quality of the grey surface, which was ground by hand under ordinary workshop conditions.

As a check on the balancing method, the curvature produced by the bending moment was observed, in the case of the coarse carborundum grey, by placing the polished surface on an optical flat and observing the depression of the centre by means of the interference bands thus produced. It can be shown that a bending moment of $125 \mathrm{gm}$. cm. would produce on this strip a depression of 16.8 bands, assuming for Young's Modulus the value $6 \times 10^{11}$ C.G.S. units. Experimentally the depression was found to be $15 \frac{1}{2}$ bands.

The accuracy of observing these depressions is, of course, not great, particularly in the finer greys, with consequently fewer interference bands. The depressions are interesting, nevertheless, as showing directly the effect of a grey surface on the perfection of figure of the opposite surface of optical work, which is a factor liable, on occasion, to be overlooked. As an illustration of this may be mentioned the case of a galvanometer mirror, one inch diameter, polished on one side to a radius of curvature of one metre, and subsequently reduced in thickness to about half a millimetre at the centre. This surface was finished with a fine grey, and when the radius was checked it was found to be 85 centimetres. Repolishing the back restored it to its correct radius of 100 centimetres.

In conclusion I wish to thank Messrs Adam Hilger, Ltd., by whose permission I am enabled to present these data, and at the same time I must apologise for the incompleteness of the subject matter. I am fully aware that there are many criticisms possible, for although the results, so far as they go, are accurate, the object of the experiments did not call for accurately standardised conditions, nor did they justify a complete investigation of the effects of varying those conditions. I present the paper in the hope that it may prove of interest in the light of Mr F. W. Preston's paper on "The Structure of Abraded Glass Surfaces."

\section{DISCUSSION}

Mr F. W. Preston (communicated): The paper seems so complete and to the point that I do not think I can add much to it immediately. The quantitative measurement of the quantity $F$, which has the dimensions of surface tension, and may be called the surface compression, marks a distinct advance. The proof that 
$F$ is a fairly definite function of the grain size of the abrasive is interesting, but it seems rather remarkable that it should be independent of the nature and other properties of the abrasive. For example, one would expect it to vary somewhat with the angularity of the abrasive. It would seem that the quality of an abrasive might almost be defined in terms of its $F$ value.

I have not hitherto been sure that it was valid to calculate $F$ as $2 M / a b$; it assumes that the stress at a point in the body of the glass varies as a linear function of the distance of the point from the grey surface. The theoretical justification for such an assumption seems to me complete, but hitherto I have not been able to make it square with the experimental evidence. This straight line law would require as a corollary that if a parallel piece of glass be greyed equally on both faces then the body of the glass is in uniform tension, the tension being equal at all points throughout the thickness. My impression has been hitherto that the double refraction was slightly greater near the grey surfaces than at a distance from them.

In view of this paper I have now repeated my observations, and am satisfied that Mr Dalladay is right and that the straight line law is valid. In particular I believe that a piece of glass greyed on both faces is in uniform tension and the strain is not localised near the faces. Our previous observations that the double refraction sometimes seems somewhat greater nearer the faces must, I think, be attributed to secondary causes, such as internal total reflection which depolarises the light a little. Mr Dalladay's view must, therefore, be accepted as a correction to the nonlinear relation more or less implied in my paper.

I am not clear just how $M$ was determined in Mr Dalladay's experiments, for the application of a neutralising bending moment will not neutralise the general extension as well as the bending moment with which it is combined. The ideal way would appear to be to grey both faces and then to apply a longitudinal compression of $2 \mathrm{Fb}$ to the specimen as a whole. This should not present any great difficulty as $F$ is of the order of 4 lbs. per inch of width. When the correct thrust was applied the whole field would become uniformly dark again between crossed nicols.

It remains to explain the shape of the curve obtained when the surface compression is plotted against coarseness of abrasive. Let the thickness of the grey layer be $t$, and let the ultimate strength of the glass be $f$. Then, by reason of the mode of formation of the grey layer, it follows that the stress there must approximate to $f^{*}$. Then if $f$ is constant throughout the grey layer, $F=f t$.

Further, if the principle of similarity were valid in such cases, $t$ would be proportional to the grain size of the abrasive. Thus $F=k f D$, where $D$ is the diameter of the abrasive grain. Thus if $f$ were a constant independent of $t$, the compression $F$ would be proportional to the grain size. It is, however, well known that very small particles of matter, very fine wires, and so on, have a strength much greater than the principle of similarity would suggest; this may account for the departure from a straight line law for this graph.

On the other hand, the degree of accuracy claimed for the measurements of $M$, and the inevitable errors involved in the determination of grain size $D$, seem to

$$
\text { * See also Twyman, Proc. Opt. Conv. (1905), p. } 52 .
$$


be such as do not definitely prove that at first the thrust increases in linear proportion, with grain size. A law of the form $F=210 D^{\cdot 33}$ might almost equally well fit the case.

Mr T. Smith asked whether the value of Young's Modulus used was that for bulk or for surface layers, and whether the effects due to the greyed rim of the lenses extended sufficiently far to affect, for example, the aberrations of a microscope objective.

Mr H. S. Ryland said that in proof plane work colour changes sometimes took place which could not be explained by temperature variations. The example of the galvanometer mirror mentioned in the paper probably afforded a satisfactory explanation.

Mr F. Twyman (partly communicated): The value adopted for Young's Modulus was taken from published tables, and was not determined for the actual sample used.

I have never been able to find that the resolving power of an echelon grating is affected by the presence of greyed edges. The double refraction only amounts to a small fraction of a wave length, and I do not think, therefore, that the definition of a microscope objective would be affected by the greyed edges.

Mr Preston has kindly associated my name with the effect of strain observed in glass which has ground surfaces. Mr Preston has also pointed out to me in correspondence that I did not either very clearly, or, as I think now, even correctly describe its details.

I wish, therefore, now to state that I am in entire agreement with Mr Dalladay as to the nature of the distribution of stress in the cases considered, and indeed have been in entire accord with him on this subject throughout the course of the experiments, with the progress of which I was continuously in touch.

Author's reply (communicated): Mr Preston has objected that the general extension would cause an error in determining the balancing point. The point of balance chosen was on the neutral axis and, as I have pointed out in the paper, the errors produced above and below the axis are of opposite sense, and therefore cause no bias in either direction.

The ideal way would certainly appear to be to apply a uniform longitudinal compression to a strip greyed on both faces. It has, however, been found by others to be an exceedingly difficult matter to apply an absolutely even compression of this kind; so much so, that the method has been abandoned whenever possible. The expenditure of time and the fitting up of the necessary apparatus were not justified by the objects of the experiments, hence the simple method adopted.

With regard to the errors involved in measuring the grain size, the abrasives used were rendered very uniform in size by elutriation, and their mean diameters measured microscopically. The errors resulting from this cause, are, therefore, small. 